\title{
Is it possible to reverse the epidemics of unnecessary cesarean sections?
}

For thousands of years, beginning in the $19^{\text {th }}$ century, the surgical technique of cesarean section was started. Was used as a last resource by obstetricians in life-threatening situations for the mothers. Therefore, the surgery was done exceptionally; its use started increasing progressively in the $20^{\text {th }}$ century. Up until World War II, data from several hospitals in developed countries showed that the percentage of elective cesarean sections was not more than 3-4\%.

Among other aspects, it is worth noting that they were cautiously considered only if warranted because a cesarean section itself increased maternal mortality significantly.

Later, in the 1950s, the advances in medicine markedly reduced such potentially serious risks for the mother. After such advances, the rate of elective cesarean sections increased progressively until reaching $10-15 \%$. This rate remained consistent for many years and was accepted by the World Health Organization, which considered it an adequate action to reduce certain severe disorders that may occur during vaginal delivery. While maintaining such rate, fetal and newborn morbidity and mortality were reduced.

I started my training in Neonatology when I was accepted as part of the residency program at Maternidad Sardá, in Buenos Aires, in 1968. Among the memories I treasure from those wonderful years, I remember that the rate of elective cesarean sections for evident risks ranged between $10 \%$ and $12 \%$. Later, in 1977, I started working at Hospital Italiano de Buenos Aires, and the rate there ranged between $13 \%$ and $15 \%$.

These ranges remained consistent for several years and perinatal outcomes continued improving, both in the field of obstetrics and neonatal intensive care, and this helped to the development of neonatology, an incipient but major discipline in the field of pediatrics, and its critical improvement of term and preterm care with a progressive reduction in morbidity and mortality.

However, unfortunately this did not last much longer and around the end of the 1980s, several aspects of medicine declined. Most of all, this affected patient care and resulted in increasing health care-related problems.

It is worth noting that one of the greatest problems in perinatal medicine was the marked progressive increase in the number of unnecessary cesarean sections.

Such increase was slow at first; however, in this century, it progressed at a faster pace until reaching rates never imagined before in terms of how it affected the manner humans are born, which has always been, ever since humans have inhabited the Earth, via vaginal delivery.

During this period, medicine was invaded by commoditization, especially in the private medical sector. Obstetricians were concerned about performing a cesarean section because payment was higher than attending a vaginal delivery; and cesarean sections were even faster than waiting for hours alongside women in labor, which usually takes longer, especially in the case of primiparous mothers, as determined by nature.

This also occurred in many other countries and, for several years now, we have coined the term "cesarean section out of convenience." This is regrettably what obstetricians do, what happens in the case of medically unnecessary cesarean sections.

Currently, at most private medical facilities, the rate of unnecessary cesarean sections is over $75 \%$ of births (in some, it is even higher), at least in Buenos Aires. At public hospitals, percentages are smaller but still above $30-35 \%$, i.e., one third of births.

It is perfectly clear that, if there is no reason or risk for the mother or the unborn baby during labor, performing a cesarean section is exclusively up to the obstetrician's desire, contrary to what should be done in pursuit of a correct and honest practice of medicine.

Multiple studies have pointed out the evident risks involved by unnecessary cesarean sections to both the mother and the newborn baby. In the first years of the century, Villar et al. ${ }^{1}$ published a multicenter study carried out in eight Latin American countries that included 97000 births. The mean number of cesarean sections was $33 \%(24-43 \%)$, and even back then, the rate was even higher at private facilities $(51 \%)$. Birth by cesarean section was significantly associated with a higher severe maternal morbidity and mortality due to postpartum antibiotic therapy. The larger number of cesarean sections was also associated with an increase in fetal mortality and a higher number of newborn infants admitted to the neonatal intensive care unit. Likewise, cesarean sections in preterm births increased neonatal mortality by $10-20 \%$.

One year later, the group led by Villar published a cohort study conducted in 420 Latin American hospitals. ${ }^{2}$ The main outcomes of that 
study indicated that mothers with a cesarean section had a higher significant risk for severe maternal morbidity compared to those with a vaginal delivery (odds ratio [OR]: 2.0, $95 \%$ confidence interval [CI]: 1.6-2.5 for cesarean section).

Several studies have found varying risks, especially in relation to different breathing disorders in the first minutes and hours of a newborn infant's life. Branco de Almeida et al. ${ }^{3}$ observed 2087 infants born via a non-urgent cesarean section and 4842 infants born via vaginal delivery and noted that anesthesia used for the cesarean section increased the risk for bag-mask ventilation (OR: 1.42, 95 \% CI: 1.071.89 ) as adjusted for the number of pregnancies, maternal hypertension, and birth weight. A study conducted in Argentina ${ }^{4}$ assessed morbidity in term vaginally-born (1120) or cesarean-born (901) infants. The greatest neonatal respiratory morbidity was observed in infants born at 37 and 38 weeks of gestation via a cesarean section (cesarean section $7.4 \%$ versus vaginal delivery $2.1 \%$; relative risk [RR]: 3.5; $95 \%$ CI: 1.5-8.1). Likewise, $68 \%$ of mothers with a cesarean section stated moderate to severe postpartum pain, compared to $36 \%$ of those with a vaginal delivery (RR: 1.9; $95 \%$ CI: 1.7-2.1). The rate of exclusive breastfeeding at the time of discharge was smaller among mothers with a cesarean section (90\% versus $96 \%$; RR: 0.94; 95 \% CI: 0.92-0.96).

Finn et al..$^{5}$ assessed respiratory rate, respiratory volume, and carbon dioxide. Values increased significantly in cesarean-born infants and an association was established with the presence of transient tachypnea.

In recent years, different proposals have been made to reduce the huge cesarean section rate. A recent article ${ }^{6}$ has provided an update on which would be the best treatment to reduce the need for cesarean sections. It mostly focused on research in the use of high doses of oxytocin to reduce frequent labor delays in nulliparous mothers during vaginal delivery. Although studies are scarce yet, they point out three ongoing clinical trials that compare low and high doses to reduce delays during the first stage of labor in nulliparous mothers. There is consensus about the fact that those studies may very likely provide evidence on the safety and effectiveness of high doses of oxytocin to reduce labor and delivery duration, thus achieving a spontaneous vaginal birth. This way, there would be no excuse to perform a cesarean section in a nulliparous woman because, other than the risks mentioned here, it leads to have any subsequent baby also via a cesarean section, which is common for the patients of most obstetricians.
McClelland et al. ${ }^{7}$ conducted a study to identify the variability-associated factors in the rate of cesarean sections at a single site and observed a broad range of cesarean section rates among obstetricians.

Finally, here I will briefly discuss the ethical aspects, which are always critical in medical practice so that our actions are patient-oriented. To this end, we should adhere to the beneficence and the nonmaleficence principles that guide our actions towards the patient's benefit. As I have mentioned above, an inadequate behavior leading to potentially severe risks for the mother and the newborn infant takes place every time an unnecessary cesarean section is performed, usually for the convenience of health care providers.This results in a clash between the physician's and the patient's interests, which is undoubtedly ethically wrong.

The reality we are facing in relation to the indication of a cesarean section that is unnecessary and for convenience purposes only is not legal or ethical; therefore, we should strongly advocate for a reduction in such high cesarean section rates, which are away from ancient medical ethical principles.

José María Ceriani Cernadas, M.D. Editor

http:/ / dx.doi.org/10.5546/ aap.2019.eng.66

To cite: Ceriani Cernadas JM. Is it possible to reverse the epidemics of unnecessary cesarean sections? Arch Argent Pediatr 2019;117(2):66-67.

\section{REFERENCES}

1. VillarJ, ValladaresE, WojdylaD,ZavaletaN,etal.Caesarean delivery rates and pregnancy outcomes: the 2005 WHO global Survey on maternal and perinatal health in Latin America. Lancet. 2006;367(9525):1819-29.

2. Villar J, Carroli G, Zavaleta N, Donner A, et al. Maternal and neonatal individuals risks and benefits associated with caesarean delivery: multicentre prospective study. BMJ. 2007;335:1025-35.

3. de Almeida MF, Guinsburg R, da Costa JO, Anchieta LM, et al. Non-urgent caesarean delivery increases the need for ventilation at birth in term newborn infants. Arch Dis Child Fetal Neonatal Ed. 2010;95(5):F326-30.

4. Ceriani Cernadas JM, Mariani G, Pardo A, Aguirre A, et al. Nacimiento por cesárea al término en embarazos de bajo riesgo: efectos sobre la morbilidad neonatal. Arch Argent Pediatr. 2010;108(1):17-23.

5. Finn D, De Meulemeester J, Dann L, Herlihy I, et al. Respiratory adaptation in term infants following elective caesarean section. Arch Dis Child Fetal Neonatal Ed. 2018 Sep;103(5):F417-F421.

6. Kenyon S, Sanders J, Middleton L, Johnston T. What is the best treatment to reduce the need for caesarean section in nulliparous women at term with delayed first stage of labour? BMJ. 2017;357:j2469.

7. McClelland S, Gorfinkle N, Arslan AA, Benedetto-Anzai MT, et al. Factors associated with cesarean delivery rates: a single-institution experience. Matern Health Neonatol Perinatol. 2017;3:8. 